\title{
Relic abundance of $\mathrm{MeV}$ millicharged particles
}

\author{
A.D. Dolgov ${ }^{1,2,3, * \text { and A.S. Rudenko }}{ }^{1,4, \oplus}$ \\ ${ }^{1}$ Novosibirsk State University, Novosibirsk, 630090, Russia \\ ${ }^{2}$ Institute for Theoretical and Experimental Physics, Moscow, 117218, Russia \\ ${ }^{3}$ Dipartimento di Fisica, Università degli Studi di Ferrara, I-44100 Ferrara, Italy \\ ${ }^{4}$ Budker Institute of Nuclear Physics, Novosibirsk, 630090, Russia
}

\begin{abstract}
The relic abundance of light millicharged particles (MCPs) with the electric charge $e^{\prime}=5 \cdot 10^{-5} e$ and with the mass slightly below or above the electron mass is calculated. The abundance depends on the mass ratio $\eta=m_{X} / m_{e}$ and for $\eta<1$ can be high enough to allow MCPs to be the cosmological dark matter or to make a noticeable contribution to it. On the other hand, for $\eta \gtrsim 1$ the cosmological energy density of MCPs can be quite low, $\Omega_{X} h_{0}^{2} \approx 0.02$ for scalar MCPs, and $\Omega_{X} h_{0}^{2} \approx 0.001$ for spin $1 / 2$ fermions. But even the lowest value of $\Omega_{X} h_{0}^{2}$ is in tension with several existing limits on the MCP abundances and parameters. However, these limits have been derived under some natural or reasonable assumptions on the properties of MCPs. If these assumptions are relaxed, a patch in the mass-charge plot of MCPs may appear, permitting them to be dark matter particles.
\end{abstract}

\section{INTRODUCTION}

Millicharged particles (which we will denote either as MCP or $X$ ) are hypothetical particles with an electric charge $e^{\prime}=\epsilon e$, which is much smaller than the elementary charge $e$, i.e. $\epsilon \ll 1$. The possibility of existence of such particles was suggested many years ago by different authors, e.g. in [1] in connection with a possible nonconservation of the electric charge, and in 2] in the model with a second $U(1)$ gauge field, the "paraphoton". Later in [3] particles with $\epsilon \ll 1$ were considered as candidates for dark matter. Since then millicharged particles were widely discussed in the literature, and various constraints on their parameters (mass and charge) were obtained from laboratory and accelerator experiments as well as from astrophysical and cosmological considerations. The plots with the excluded regions of mass-charge parameter space one can find e.g. in [4 10]. The recent limits allow only for a minor cosmological fraction of MCPs, the best one up to now is $\Omega_{X} h_{0}^{2}<0.001$ (95\% CL) 11].

However, all the limits are derived under some minimal assumptions on the MCP properties. The only thing which is taken for sure is the MCP electromagnetic interaction with a tiny electric charge. By default all (or almost all) other possible interactions of MCPs are neglected. We will not use these assumptions and take instead the maximum freedom principle, i.e. assume that anything which is not explicitly excluded is permitted. If this is the case, then MCPs can make up the whole cosmological dark matter or be a considerable fraction of it.

Our paper is organized as follows. In Sect. [II we explain in more detail motivation of the study. In Sect. III we discuss the kinetic equation which describes the number of $X$-particles in expanding universe and analytically calculate their cosmological abundance for the case of $m_{X}$ slightly above and slightly below $m_{e}=0.511 \mathrm{MeV}$ (subthreshold annihilation). In Sect. IV] we present the results of the numerical computations. Finally, in Sect. $\mathrm{V}$ we conclude.

\section{MOTIVATION}

The idea that millicharged particles constitute a part of dark matter is quite intriguing. For instance, it was proposed recently [12] that the mystery of the origin of galactic magnetic fields could be solved if one assumed that these fields were created due to interaction between electrons and millicharged dark matter particles.

The particles much lighter than $1 \mathrm{MeV}$ and with the charge, $\epsilon \gtrsim 10^{-9}$, are supposed to be excluded by their impact on Big Bang Nucleosynthesis (BBN) [8]. However, an agreement with observations can be restored if the cosmological lepton asymmetry is non-vanishing [13]. Nevertheless, we will not consider here very light millicharged particles with $m_{X} \ll 1 \mathrm{MeV}$.

The limits on $\epsilon$ are obtained also from invisible decays of orthopositronium. Such limits are applied only for $m_{X}<m_{e}$ and constitute $\epsilon<3.4 \cdot 10^{-5}$ for $m_{X}<<m_{e}$ [10]. However, they are quite weak for $m_{X} \lesssim m_{e}$, because the decay probability is proportional to $\left(1-m_{X}^{2} / m_{e}^{2}\right)^{n / 2}$ (here $n=1$ for spin $1 / 2 X$-particles and $n=3$ for spin 0) [14], and for $m_{X} \geq m_{e}$ no constraint on $\epsilon$ can be derived at all.

\footnotetext{
* dolgov@fe.infn.it

† a.s.rudenko@inp.nsk.su
} 
The experiment uniquely suited to the detection of millicharged particles was performed at SLAC [7], where the following bounds $\left(95 \% \mathrm{CL}\right.$ ) were obtained: $\epsilon<2.0 \cdot 10^{-5}$ for $m_{X}=0.1 \mathrm{MeV}$, and $\epsilon<4.1 \cdot 10^{-5}$ for $m_{X}=1$ $\mathrm{MeV}$. However, without the assumption of linearity of the scintillator for very small energy depositions, the bounds are a factor of 2 less stringent [7]. Roughly speaking, according to this experiment the electric charge of MCPs is bounded from above by $\epsilon \lesssim 5 \cdot 10^{-5}$ for $m_{X} \sim m_{e}$. The SLAC bounds are valid if MCPs have sufficiently weak interaction with the usual matter to propagate 110 meters of the sandstone between the source and detector. This is true if the only interaction of MCPs is the milli-electromagnetic one. New stronger interactions could destroy the bound. Some related references can be found in the list [15], where restrictive bounds are derived but probably more exotic options still remain open.

There is also the region $10^{-9} \lesssim \epsilon \lesssim 10^{-7}, m_{X} \lesssim 5 \mathrm{MeV}$ which is excluded by consideration of the energy-loss rate of the Supernova 1987A [8], but we are interested here in larger allowed values of $\epsilon$.

Besides the discussed results, the millicharged particles with $\epsilon \lesssim 5 \cdot 10^{-5}$ and $m_{X} \sim m_{e}$ are reported to be excluded by the limits on their relic abundance, $\Omega_{X} h_{0}^{2}$. The corresponding exclusion plot can be found e.g. in [9]. Therein the limits were obtained using the Lee-Weinberg formula [16] for the relic abundances, precise cosmic microwave background (CMB) data from WMAP, and the standard BBN scenario. However, details of calculation, namely how the bound on $\Omega_{X} h_{0}^{2}$ was translated into the constraints on mass and charge of $X$-particles, were not given there. Therefore, we believe that it is necessary to revisit these bounds with more accuracy. So, in this paper we calculate carefully the relic abundance of millicharged particles with masses not very different from the electron mass, $m_{X} \sim m_{e}$. We consider not only the usual case when $X$-particles can annihilate into lighter particles, but also the annihilation in $e^{+} e^{-}$"below threshold" (if $m_{X}<m_{e}$ ) which is allowed for energetic $X$-particles from the tail of their energy distribution [17-19]. To the best of our knowledge, subthreshold annihilation of MCPs was not studied before.

As it has been mentioned above, the most stringent bound is obtained from the analysis of the angular fluctuation spectrum of CMB performed in [11]. An essential point in the derivation of this bound is the assumption that MCPs and protons with electrons are strongly coupled to each other, so they oscillate as a unique substance creating the acoustic oscillations of the photon temperature. So the shape of the angular spectrum is determined by the sum of the cosmological densities of protons and MCPs. Separate measurement of the diffusion (Silk) damping of high multipoles allows to separate the contribution of protons and MCPs and to obtain the record bound on the cosmological abundance of MCPs. The assumption of a sufficiently strong coupling between protons and MCPs is based on the estimate of the Coulomb interactions between them. This estimate is valid if the temperatures of the protons and MCPs are the same and equal to the temperature of the CMB photons. However, one can imagine a scenario, e.g. with some new particles interacting with MCPs, when the MCP temperature could be noticeably higher than $T_{\gamma}$. In this case the Coulomb coupling drops down, MCPs do not participate in the proton acoustic oscillations, and the bound is destroyed. This scenario will be studied elsewhere.

\section{BOLTZMANN EQUATION}

When the universe was hot enough, millicharged particles were in thermal equilibrium, if $\epsilon$ was not vanishingly tiny. For example, for the temperatures larger than $m_{X}$, equilibrium with respect to the elastic MCP scattering off electrons, as well as $X \bar{X}$ annihilation into $e^{+} e^{-}$pairs, was established at $T \lesssim \alpha^{2} \epsilon^{2} m_{P l} \sim 10^{5}\left(\epsilon / 10^{-5}\right)^{2}$ $\mathrm{GeV}$. However, the universe was expanding and after the moment when the annihilation rate of $X \bar{X}$ pairs became smaller than Hubble parameter, $\Gamma_{a n n} \lesssim H$, due to the Boltzmann suppression of their number density at $T<m_{X}$, the $X$-particle annihilation practically stopped and their number became constant in comoving volume. This phenomenon is called freeze-out.

The derivation of Boltzmann (kinetic) equation which describes the evolution of particle number density before and after decoupling is discussed in detail in [17, 20]. Here we briefly remind this derivation and assumptions under which it is valid.

Let us assume there are no other particles beyond the Standard Model (SM) ones, except MCPs, which can be scalars or fermions. Such $X$-particles are supposed to be stable and interacting directly only with photons and via photons with other charged particles of SM. It is also implicitly assumed that $\epsilon$, though small, but still is large enough, so the specified below conditions are fulfilled.

The temperature of freeze-out, $T_{f}$, should be much smaller than $m_{X}$, otherwise MCPs would overclose the universe. For $m_{X} \sim m_{e}$ the production and annihilation of MCPs proceeded mainly through the reaction $X \bar{X} \leftrightarrow e^{+} e^{-}$, the cross-section of which at low energies is $\sim \epsilon^{2} \alpha^{2} / m_{X}^{2}$ (see Fig. (1). Other inelastic processes involving $X$-particles are inessential at such temperatures, e.g. the cross-section of two-photon annihilation $X \bar{X} \rightarrow \gamma \gamma$ is heavily suppressed as $\epsilon^{4} \alpha^{2}$, and the plasmon decay $\gamma_{P} \rightarrow X \bar{X}$ is also ineffective for $m_{X} \sim m_{e}$ (plasmon decay is operative at $T \gtrsim 10 \mathrm{MeV}$ since the plasmon "mass" (plasma frequency) $\omega_{P} \sim 0.1 T$ must be $\left.\gtrsim 2 m_{X}\right)$ [21]. One can consider also the three-particle annihilation $X \bar{X} e^{ \pm} \rightarrow e^{ \pm} \gamma$, the cross-section of which is suppressed only as $\epsilon^{2} \alpha^{3}$, but it has an additional phase space suppression with respect to the two-body channel 
$X \bar{X} \rightarrow e^{+} e^{-}$. However, such three-particle reaction may be essential for temperatures and masses of MCPs lower than ones considered here, when ordinary annihilation $X \bar{X} \rightarrow e^{+} e^{-}$is heavily suppressed.

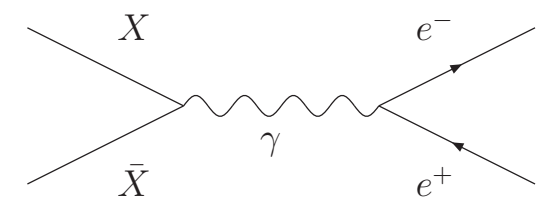

FIG. 1. The Feynman diagram for $X \bar{X} \rightarrow e^{+} e^{-}$annihilation

It is usually assumed that kinetic equilibrium of $X$-particles was maintained even after freezing of their annihilation, i.e. at temperatures $T \lesssim m_{X}$. The equilibrium remained while the rate of elastic scattering $X+e^{-} \rightarrow X+e^{-}$was much larger than the rate of the universe expansion, $H$. If the asymmetry between particles and antiparticles was not too high, the particle occupation numbers were small, $f \ll 1$, and thus at this period $X$-particles, electrons, and positrons obeyed the Boltzmann statistics and were described by the equilibrium distribution functions, $f_{i}^{e q}=\exp \left[-\left(E_{i}-\mu_{i}\right) / T\right]$, with $E_{i}$ and $\mu_{i}$ being respectively the energy and the chemical potential of $i$-th sort of particles.

Chemical potentials are introduced to describe a difference between the number densities of particles and antiparticles in thermal equilibrium. The evolution of $\mu_{j}$ is governed by inelastic reactions and in full equilibrium they satisfy the condition $\mu+\bar{\mu}=0$, where $\bar{\mu}$ is the chemical potential of antiparticles. If the chemical equilibrium is not maintained, the above condition is not necessarily fulfilled and chemical potentials may be non-zero even in absence of particle-antiparticle asymmetry. Usually it is assumed that the number density of $X$-particles is equal to that of antiparticles, $n_{X}=n_{\bar{X}}$. When the annihilation of $X \bar{X}$ was frozen but kinetic equilibrium is maintained, the particle and antiparticle distributions have the same form $f=\exp [(\mu-E) / T]$ with $\mu=\bar{\mu}$. In this case $\mu$ is often called effective chemical potential, which is usually a function of time but not of the particle energy. Correspondingly the distribution can be presented as

$$
f=C(t) f^{(e q)} \equiv C(t) \exp (-E / T)
$$

We also assume that $T$-invariance holds, therefore amplitudes squared and summed over spins are equal for direct and reverse reactions, $\sum_{s}\left|A_{a \rightarrow b}\right|^{2}=\sum_{s}\left|A_{b \rightarrow a}\right|^{2}$, with an evident change of the signs of velocities of the participating particles.

Finally, under all these assumptions the Boltzmann equation for $X$-particles in FLRW metric can be written as

$$
\dot{n}_{X}+3 H n_{X}=-<\sigma v>\left(n_{X}^{2}-n_{X, e q}^{2}\right) .
$$

Here $n_{X}$ is the number density of $X$-particles, $n_{X, e q}$ is the equilibrium one, i.e. having vanishing chemical potential, $H=\dot{a} / a$ is the Hubble parameter ( $a$ is the cosmological scale factor), and $\langle\sigma v\rangle$ is thermally averaged cross-section of process $X \bar{X} \rightarrow e^{+} e^{-}$times the Møller velocity.

Following [17] one can obtain that

$$
<\sigma v>=\frac{1}{8 m_{X}^{4} T K_{2}^{2}\left(m_{X} / T\right)} \int_{\max \left(4 m_{X}^{2}, 4 m_{e}^{2}\right)}^{\infty} \sqrt{s}\left(s-4 m_{X}^{2}\right) \sigma(s) K_{1}\left(\frac{\sqrt{s}}{T}\right) d s,
$$

where the cross-section $\sigma\left(X \bar{X} \rightarrow e^{+} e^{-}\right)$is summed over final and averaged over initial spins, $K_{i}$ are the modified Bessel functions of second kind and order $i$. Note that the cases $m_{X} \leq m_{e}$ and $m_{X} \geq m_{e}$ differ from each other only in lower limit of integral (3.3).

Instead of $n_{X}(t)$ let us introduce the dimensionless quantity $Y(\xi)=n_{X} / s$. Here $\xi=m_{X} / T$ and $s=$ $g_{* s}\left(2 \pi^{2} / 45\right) T^{3}$ is the entropy density (it should not be confused with the Mandelstam variable $s$ ), where $g_{* s}=$ 3.94 is the effective number of relativistic species in the entropy density for $T \ll m_{e}$, which includes photons and three species of massless neutrinos. The function $Y(\xi)$ is very convenient because of the relation $\dot{s}+3 H s=0$, which means that total entropy in comoving volume is conserved, sa $a^{3}=$ const. Moreover, $Y(\xi)$ has a simple physical meaning, it is proportional to the total number of $X$-particles, $Y=n_{X} / s \sim n_{X} a^{3}=N_{X}$.

In radiation-dominated universe $(T \gtrsim 1 \mathrm{eV})$ the energy density is

$$
\rho=\frac{3}{32 \pi} \frac{m_{P l}^{2}}{t^{2}}=g_{*} \frac{\pi^{2}}{30} T^{4}
$$

where $m_{P l} \approx 1.22 \cdot 10^{19} \mathrm{GeV}$ is the Planck mass, $g_{*}=3.38$ is the effective number of relativistic species in the cosmological energy density for $T \ll m_{e}$. The relation (3.4) shows that temperature $T$ behaves like $1 / \sqrt{t}$. Now it is straightforward to change variable $t$ to $\xi=m_{X} / T$. 
The Boltzmann equation (3.2) then becomes

$$
\frac{d Y}{d \xi}=-\sqrt{\frac{45}{4 \pi^{3} g_{*}}} \frac{m_{P l}}{m_{X}^{2}} \xi s<\sigma v>\left(Y^{2}-Y_{e q}^{2}\right),
$$

where $Y_{e q}=n_{X, e q} / s$, and $n_{X, e q}=\left(g_{X} / 2 \pi^{2}\right) K_{2}\left(m_{X} / T\right) m_{X}^{2} T$ [17] (here $g_{X}$ is the number of spin degrees of freedom for $X$-particles, $g_{X}=1$ for scalars and $g_{X}=2$ for spin $1 / 2$ fermions).

I. When $X$-particles are scalars, the cross-section of $X \bar{X} \rightarrow e^{+} e^{-}$annihilation for non-identical $X$ and $\bar{X}$ is equal to:

$$
\sigma\left(X \bar{X} \rightarrow e^{+} e^{-}\right)=\frac{4 \pi}{3} \alpha^{2} \epsilon^{2} \frac{1}{s} \sqrt{1-\frac{4 m_{e}^{2}}{s}} \sqrt{1-\frac{4 m_{X}^{2}}{s}}\left(1+\frac{2 m_{e}^{2}}{s}\right) .
$$

The factor $\sqrt{1-4 m_{e}^{2} / s}$ comes as usually from the phase space of the final particles and the factor $\sqrt{1-4 m_{X}^{2} / s}$ originates here from the initial c.m. velocity related to the scalar particles annihilation in $P$-wave. Therefore, this cross-section vanishes for $s=4 m_{X}^{2}$ and/or $s=4 m_{e}^{2}$.

Substituting (3.6) into (3.3) one finds

$$
<\sigma v>=\frac{4 \pi}{3} \alpha^{2} \epsilon^{2} \frac{1}{m_{X}^{2}} \frac{\xi}{\eta^{3} K_{2}^{2}(\xi)} I_{s}(\xi, \eta),
$$

where

$$
I_{s}(\xi, \eta)=\int_{\max \left(1, \eta^{2}\right)}^{\infty} \sqrt{x-1}\left(1-\frac{\eta^{2}}{x}\right)^{3 / 2}\left(1+\frac{1}{2 x}\right) K_{1}\left(\frac{2 \xi \sqrt{x}}{\eta}\right) d x
$$

with $x=s / 4 m_{e}^{2}, \eta=m_{X} / m_{e}$.

II. When $X$-particles are spin $1 / 2$ fermions,

$$
\sigma\left(X \bar{X} \rightarrow e^{+} e^{-}\right)=\frac{4 \pi}{3} \alpha^{2} \epsilon^{2} \frac{1}{s} \frac{\sqrt{1-4 m_{e}^{2} / s}}{\sqrt{1-4 m_{X}^{2} / s}}\left(1+\frac{2 m_{e}^{2}}{s}+\frac{2 m_{X}^{2}}{s}+\frac{4 m_{X}^{2} m_{e}^{2}}{s^{2}}\right) .
$$

For $m_{X}=m_{e}=m$ this cross-section is a finite constant, $\sigma=3 \pi \alpha^{2} \epsilon^{2} / 4 m^{2}$ at threshold $s=4 m^{2}$.

Substituting (3.9) into (3.3) one has

$$
<\sigma v>=\frac{4 \pi}{3} \alpha^{2} \epsilon^{2} \frac{1}{m_{X}^{2}} \frac{\xi}{\eta^{3} K_{2}^{2}(\xi)} I_{f}(\xi, \eta),
$$

where

$$
I_{f}(\xi, \eta)=\int_{\max \left(1, \eta^{2}\right)}^{\infty} \sqrt{x-1}\left(1-\frac{\eta^{2}}{x}\right)^{1 / 2}\left(1+\frac{1}{2 x}+\frac{\eta^{2}}{2 x}+\frac{\eta^{2}}{4 x^{2}}\right) K_{1}\left(\frac{2 \xi \sqrt{x}}{\eta}\right) d x .
$$

Finally, the Boltzmann equation takes the following form

$$
\frac{d Y}{d \xi}=-\frac{\epsilon_{5}^{2}}{\eta^{4}} I(\xi, \eta)\left(a \frac{Y^{2}}{\xi K_{2}^{2}(\xi)}-b \xi^{3}\right)
$$

where $\epsilon_{5}=10^{5} \cdot \epsilon ; I(\xi, \eta)=I_{s}$ when $X$ is a scalar, and $I(\xi, \eta)=I_{f}$ when $X$ is a spin $1 / 2$ fermion; $a$ and $b$ are the numerical constants:

$$
\begin{gathered}
a=10^{-10} \cdot \frac{4 \sqrt{5} \pi^{3 / 2} \alpha^{2}}{45} \frac{m_{P l}}{m_{e}} \frac{g_{* s}}{\sqrt{g_{*}}} \approx 3.0 \cdot 10^{8}, \\
b=10^{-10} \cdot \frac{45 \sqrt{5} \alpha^{2}}{4 \pi^{13 / 2}} \frac{m_{P l}}{m_{e}} \frac{g_{X}^{2}}{g_{* s} \sqrt{g_{*}}} \approx 2.6 \cdot 10^{5} \cdot g_{X}^{2} .
\end{gathered}
$$

As it is mentioned above, due to the universe expansion the reaction $X \bar{X} \rightarrow e^{+} e^{-}$eventually "freezes out". After that the number of stable $X$-particles remains constant in the comoving volume, and nowadays their energy density tends to:

$$
\rho_{X, 0}=m_{X} \cdot n_{X, 0}=m_{e} \eta \cdot Y_{0} s_{0}
$$


Here the present-day values are marked with the index $0, s_{0} \approx 2.9 \cdot 10^{3} \mathrm{~cm}^{-3}$ is the present entropy density, and we take into account that massive $X$-particles are nonrelativistic today.

The contribution of such $X$-particles to the cosmological energy density constitutes

$$
\Omega_{X} h_{0}^{2}=\frac{\rho_{X, 0}}{\rho_{c}} h_{0}^{2} \simeq 1.4 \cdot 10^{5} \eta Y_{0},
$$

where $\rho_{c} \approx 1.88 \cdot 10^{-29} h_{0}^{2} \mathrm{~g} / \mathrm{cm}^{3}$ is the critical density of universe.

\section{NUMERICAL CALCULATION}

Here we solve the Boltzmann equation (3.12) numerically in order to calculate $Y_{0}$ and accordingly $\Omega_{X} h_{0}^{2}$. Obviously, the weaker interaction of $X$-particles is, the larger number of them remains after their decoupling, i.e. a smaller $\epsilon_{5}$ corresponds to a larger $\Omega_{X} h_{0}^{2}$. We use throughout the calculation the value $\epsilon_{5}=5$, which corresponds the upper bound on $\epsilon$ obtained at SLAC [7], though as we mentioned above this limit may be questioned if some anomalous interactions of $X$ are effective. Since the value of the electric charge of $X$-particles is fixed now, there remains only one free parameter, $\eta=m_{X} / m_{e}$.

The plot of function $Y(\xi)$ for scalar $X$ and $m_{X}=m_{e}$ is presented in Fig. 2. The picture for another mass and/or spin of $X$ would be similar: when the temperature is high enough, the function $Y(\xi)$ is close to the equilibrium one, $Y_{e q}(\xi)$, but when the temperature drops below $\sim m_{X} / 20$ the equilibrium is upset and $Y(\xi)$ tends to the constant value, $Y_{0}$.

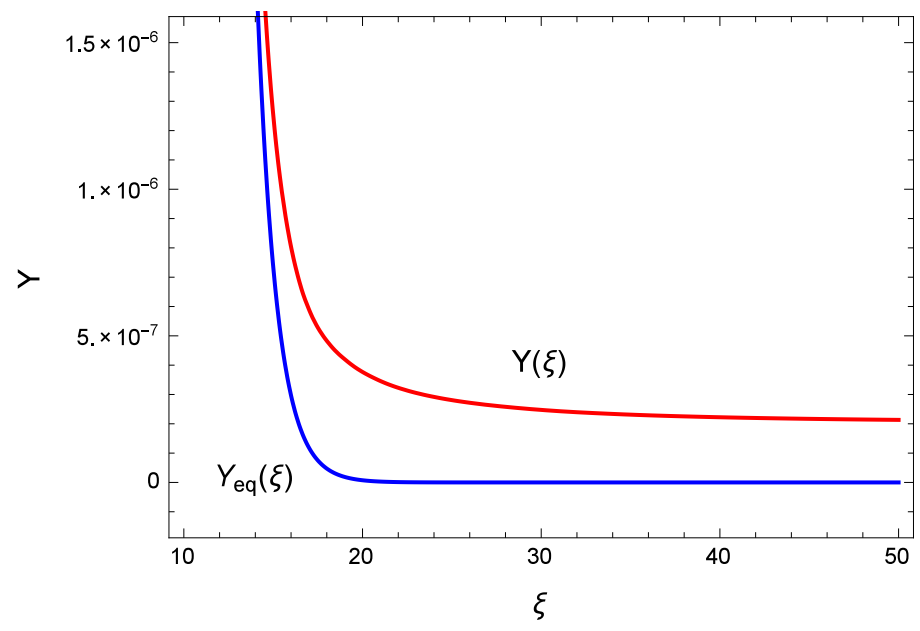

FIG. 2. The red curve shows the numerical solution $Y(\xi)$ of Boltzmann equation (3.12) for scalar millicharged particles and parameters $\epsilon_{5}=5, \eta=1\left(m_{X}=m_{e}\right)$. The blue one is the equilibrium function, $Y_{e q}(\xi)$.

Calculating numerically $Y_{0}$ for different values of parameter $\eta$ and using Eq. (3.16) one can find $\Omega_{X} h_{0}^{2}$ for $X$-particles, both scalars and fermions. The corresponding results are presented in Fig. 3 ,
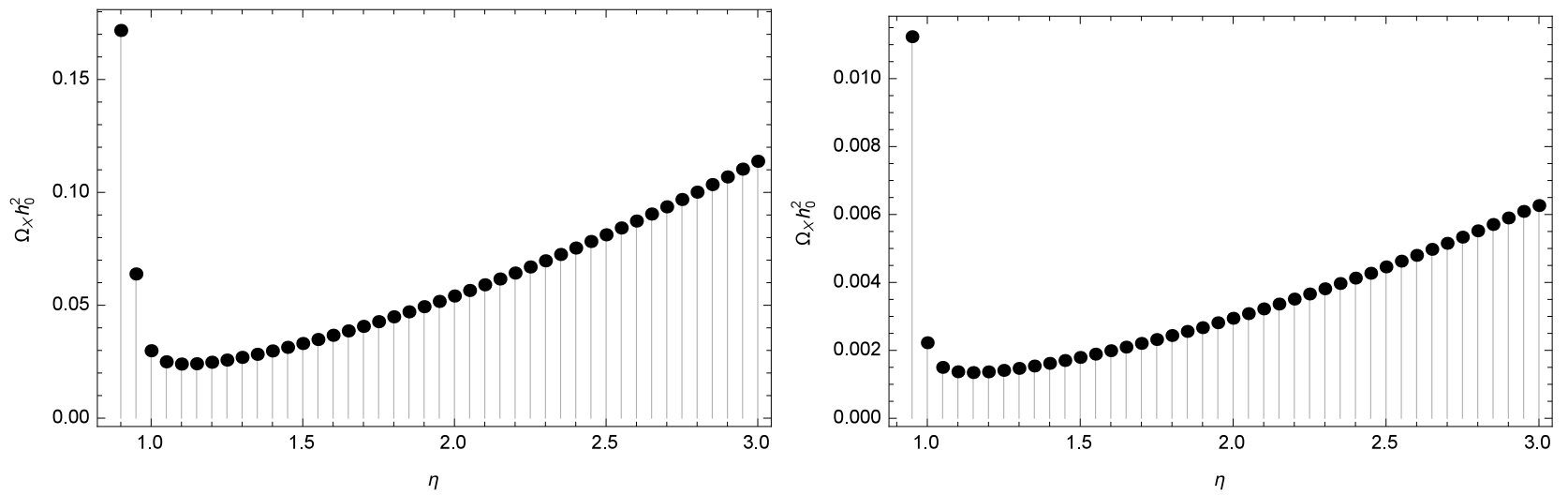

FIG. 3. The relic abundance, $\Omega_{X} h_{0}^{2}$, of millicharged particles - scalars (left plot) and spin $1 / 2$ fermions (right plot), for different values of the mass ratio, $\eta=m_{X} / m_{e}$, and for fixed $\epsilon_{5}=5$. 
The large values of $\Omega_{X} h_{0}^{2}$ for small $\eta$ have the simple explanation. If annihilation $X \bar{X} \rightarrow e^{+} e^{-}$proceeds "below threshold" $\left(m_{X}<m_{e}\right)$, the smaller is $\eta$, the lower is the number of $X$-particles at the high energy tail of their distribution which have enough energy to annihilate into heavier $e^{+} e^{-}$. Therefore, $\Omega_{X} h_{0}^{2}$ grows exponentially fast for $\eta<1$, when $\eta$ decreases.

When $\eta>1$, all $X$-particles annihilate "above threshold". Therefore, $Y_{0}$ weakly depends on $\eta$ and remains almost constant, but $\Omega_{X} h_{0}^{2}$ in general slightly increases with growth of $\eta$, mainly due to the factor $\eta$ in r.h.s. of Eq. (3.16). Therefore, it turns out that at $\eta \approx 1.1$ the cosmological fraction of MCPs, $\Omega_{X} h_{0}^{2}$, takes the minimal value, when exponential decrease of function $\Omega_{X} h_{0}^{2}(\eta)$ changes to its slow growth. With rising $\eta$, the number density of MCPs behaves as $n_{X} \sim 1 /\left(\sigma_{a n n} m_{X}\right) \sim m_{X}$ and hence their energy density rises as $m_{X}^{2}$, i.e. $\Omega_{X} h_{0}^{2}$ increases like $\eta^{2}$.

The values of $\Omega_{X} h_{0}^{2}$ for fermions are much smaller than those for scalars at the same $\eta$ (see Fig. 3), because of behavior of the corresponding annihilation cross-sections: since scalars annihilate in $P$-wave, their cross-section is suppressed near the threshold by an extra power of velocity, $v \sim T / m_{X}$, while this is not the case for fermions which annihilate in $S$-wave.

\section{CONCLUSION}

We have calculated the cosmological energy density, $\Omega_{X} h_{0}^{2}$, of millicharged particles (MCPs) with masses $m_{X} \sim m_{e}$ and with the electric charge $e^{\prime}=5 \cdot 10^{-5} e$ which is the maximal value of MCP charge allowed by SLAC experiment [7]. We have found that $\Omega_{X} h_{0}^{2}$ can vary in a wide range of values, depending on the ratio $\eta=m_{X} / m_{e}$. For the subthreshold annihilation $X \bar{X} \rightarrow e^{+} e^{-}\left(m_{X}<m_{e}\right)$ it can be even as large as the observed energy density of dark matter $\sim 0.2$. On the contrary, for $m_{X} \gtrsim m_{e}$ the cosmological energy density of MCPs can be low enough, $\Omega_{X} h_{0}^{2} \approx 0.02$ for scalar MCPs, and $\Omega_{X} h_{0}^{2} \approx 0.001$ for spin $1 / 2$ fermions.

However, even the lowest value of $\Omega_{X} h_{0}^{2}$ obtained here either contradicts (for scalars) or at least is in some tension (for spin 1/2 fermions) with the most stringent CMB bound [11], $\Omega_{X} h_{0}^{2}<0.001$ (95\% CL). Therefore, it seems that in simple models millicharged particles (especially scalars) can not contribute to the dark matter.

Nevertheless, in more complicated scenarios the possibility that millicharged particles can constitute some part of dark matter still remains. In particular, the CMB bound [11] can be considerably weakened if the temperature of the relic MCPs is higher than the proton temperature near the hydrogen recombination. This exotic possibility can be realized if there exists a new long lived particle, which decays to $X \bar{X}$-pair prior to recombination and heats them up. However, for an effective heating a new stronger interaction between MCPs is necessary.

On the other hand, the SLAC bound [7] on the value of millicharge may be relaxed if new (anomalous) interactions of $X$-particles exist which could strongly diminish their mean free path in matter, but more work is necessary to check compatibility of this hypothesis with other particle physics data.

Therefore, it is still not completely forbidden that the MCPs with $m_{X} \sim m_{e}$ can be noticeable part of the cosmological dark matter. However, more detailed investigation of the suggested and other exotics is surely needed to satisfy bounds from the particle physics experiments.

\section{ACKNOWLEDGEMENTS}

We acknowledge support of the Grant of President of Russian Federation for the leading scientific Schools of Russian Federation, NSh-9022-2016.2.

[1] A.Yu. Ignatiev, V.A. Kuzmin, M.E. Shaposhnikov, Is the electric charge conserved?, Phys. Lett. B 84 (1979) 315.

[2] B. Holdom, Two U(1)'s and Epsilon Charge Shifts, Phys. Lett. B 166 (1986) 196.

[3] H. Goldberg, L.J. Hall, A New Candidate for Dark Matter, Phys. Lett. B 174 (1986) 151.

[4] M.I. Dobroliubov, A.Yu. Ignatiev, Millicharged particles, Phys. Rev. Lett. 65 (1990) 679.

[5] S. Davidson, B. Campbell, D.C. Bailey, Limits on particles of small electric charge, Phys. Rev. D 43 (1991) 2314.

[6] S. Davidson, M. Peskin, Astrophysical bounds on millicharged particles in models with a paraphoton, Phys. Rev. D 49 (1994) 2114 hep-ph/9310288.

[7] A.A.Prinz et al., Search for millicharged particles at SLAC, Phys. Rev. Lett. 81 (1998) 1175 hep-ex/9804008.

[8] S. Davidson, S. Hannestad, G. Raffelt, Updated bounds on millicharged particles, JHEP 05 (2000) 003 hep-ph/0001179.

[9] S.L. Dubovsky, D.S. Gorbunov, G.I. Rubtsov, Narrowing the window for millicharged particles by CMB anisotropy, JETP Lett. 79 (2004) 1 [Pisma Zh. Eksp. Teor. Fiz. 79 (2004) 3] hep-ph/0311189]. 
[10] A. Badertscher et al., An Improved Limit on Invisible Decays of Positronium, Phys. Rev. D 75 (2007) 032004 hep-ex/0609059.

[11] A.D. Dolgov, S.L. Dubovsky, G.I. Rubtsov, I.I. Tkachev, Constraints on millicharged particles from Planck data, Phys. Rev. D 88 (2013) 117701 arXiv:1310.2376 [hep-ph]].

[12] Z. Berezhiani, A.D. Dolgov, I.I. Tkachev, Dark matter and generation of galactic magnetic fields, Eur. Phys. J. C 73 (2013) 2620 arXiv:1307.6953 [astro-ph.CO]].

[13] Z. Berezhiani, A.D. Dolgov, I.I. Tkachev, BBN with light dark matter, JCAP 1302 (2013) 010 arXiv:1211.4937 [astro-ph.CO]].

[14] S.N. Gninenko, N.V. Krasnikov, A. Rubbia, Positronium physics beyond the standard model, Mod. Phys. Lett. A 17 (2002) 1713.

[15] M. Pospelov, Secluded U(1) below the weak scale, Phys. Rev. D 80 (2009) 095002 arXiv:0811.1030 [hep-ph]];

B. Batell, M. Pospelov, A. Ritz, Exploring Portals to a Hidden Sector Through Fixed Targets, Phys. Rev. D 80 (2009) 095024 arXiv:0906.5614 [hep-ph]];

M.D. Diamond, P. Schuster, Searching for Light Dark Matter with the SLAC Millicharge Experiment, Phys. Rev. Lett. 111 (2013) 221803 arXiv:1307.6861 [hep-ph]];

H. Vogel, J. Redondo, Dark radiation constraints on minicharged particles in models with a hidden photon, JCAP 1402 (2014) 029 arXiv:1311.2600 [hep-ph]];

B. Batell, P. deNiverville, D. McKeen, M. Pospelov, A. Ritz, Leptophobic Dark Matter at Neutrino Factories, Phys. Rev. D 90 (2014) 115014 arXiv:1405.7049 [hep-ph]];

B. Batell, R. Essig, Z. Surujon, Strong Constraints on Sub-GeV Dark Sectors from SLAC Beam Dump E137, Phys. Rev. Lett, 113 (2014) 171802 arXiv:1406.2698 [hep-ph]];

A. Fradette, M. Pospelov, J. Pradler, A. Ritz, Cosmological Constraints on Very Dark Photons, Phys. Rev. D 90 (2014) 035022 arXiv:1407.0993 [hep-ph]];

D.E. Soper, M. Spannowsky, C.J. Wallace, T.M.P. Tait, Scattering of dark particles with light mediators, Phys. Rev. D 90 (2014) 115005 arXiv:1407.2623 [hep-ph]];

R. Foot, S. Vagnozzi, Dissipative hidden sector dark matter, Phys. Rev. D 91 (2015) 023512 arXiv:1409.7174 [hep$\mathrm{ph}]$;

E. Izaguirre, I. Yavin, New window to millicharged particles at the LHC, Phys. Rev. D 92 (2015) 035014 arXiv:1506.04760 [hep-ph]];

F. Nozzoli, A balance for dark matter bound states, Astropart. Phys. 91 (2017) 22 arXiv:1608.00405 [astro-ph.IM]].

[16] Ya.B. Zeldovich, Survey of Modern Cosmology, Advances in Astronomy and Astrophysics 3 (1965) 241;

M.I. Vysotsky, A.D. Dolgov, Ya.B. Zeldovich, Cosmological Restriction on Neutral Lepton Masses, JETP Lett. 26 (1977) 188 [Pisma Zh. Eksp. Teor. Fiz. 26 (1977) 200];

B.W. Lee, S. Weinberg, Cosmological Lower Bound on Heavy Neutrino Masses, Phys. Rev. Lett. 39 (1977) 165.

[17] P. Gondolo, G. Gelmini, Cosmic abundances of stable particles: Improved analysis, Nucl. Phys. B 360 (1991) 145.

[18] K. Griest, D. Seckel, Three exceptions in the calculation of relic abundances, Phys. Rev. D 43 (1991) 3191.

[19] R.T. D'Agnolo, J.T. Ruderman, Light Dark Matter from Forbidden Channels, Phys. Rev. Lett. 115 (2015) 6, 061301 arXiv:1505.07107 [hep-ph]].

[20] E.W. Kolb, M.S. Turner, The Early Universe, Front. Phys. 69 (1990) 1.

[21] Z. Berezhiani, A. Lepidi, Cosmological bounds on the 'millicharges' of mirror particles, Phys. Lett. B 681 (2009) 276 arXiv:0810.1317 [hep-ph]]. 